International Journal of Applied Mathematics

Volume 28 No. $6 \quad 2015,703-714$

ISSN: 1311-1728 (printed version); ISSN: 1314-8060 (on-line version)

doi: http://dx.doi.org/10.12732/ijam.v28i6.5

\title{
THE SOLUTION OF TRIANGLE ELEMENT APPROXIMATION FOR 2D HELMHOLTZ EQUATIONS USING QSGS METHOD
}

\author{
Mohd Kamalrulzaman Md Akhir ${ }^{1}$, Jumat Sulaiman ${ }^{2}$ \\ ${ }^{1,3}$ Faculty of Science and Natural Resources \\ Universiti Malaysia Sabah \\ Locked Bag 2073, 88400 Kota Kinabalu, Sabah, MALAYSIA
}

\begin{abstract}
This study aims to demonstrate the efficiency of the quarter-sweep Gauss Seidel (QSGS) method using the quarter-sweep approximation equation based on a Galerkin scheme in order to solve two-dimensional Helmholtz equations. Furthermore, the basic formulations of the Full-Sweep and Half-Sweep Gauss Seidel methods, namely FSGS and HSGS respectively are also presented. The numerical results of test examples are also included in order to verify the performance of the proposed method.
\end{abstract}

AMS Subject Classification: 41A55, 45A05, 45B05

Key Words: Helmholtz equations, Galerkin scheme, triangle element, quartersweep iterations

\section{Introduction}

Helmholtz equations often appear in various applied fields, including, but not limited to, science and engineering. It is widely employed to model problems of time harmonic acoustic and electromagnetic fields, optical waveguide, acoustic wave scattering, noise reduction in silencer, water wave propagation, radar scattering and light wave propagation problems $[4,15,16]$. In solving these problems, various researchers have recommended the use of common numerical

Received: August 11, $2015 \quad$ (c) 2015 Academic Publications

${ }^{\S}$ Correspondence author 
methods such as mesh-based methods in order to formulate numerical solutions. There exist several available schemes in mesh-based methods, including finite element, finite difference, boundary element and finite volume, which are widely used to construct approximation equations in science and engineering problems: linear, nonlinear, homogeneous and inhomogeneous.

On the other hand, this study is primarily concerned with triangle element solutions based on the Galerkin scheme for solving two-dimensional Helmholtz equations. Generally, two-dimensional (2D) Helmholtz equations in the generic form are defined as follows:

$$
\frac{\partial^{2} U}{\partial x^{2}}+\frac{\partial^{2} U}{\partial y^{2}}-\alpha U=f(x, y), \quad(x, y) \in[a, b] \times[a, b]
$$

with the Dirichlet boundary conditions

$$
\begin{aligned}
& U(x, a)=g_{1}(x), \quad a \leq x \leq b, \quad U(x, b)=g_{2}(x), \quad a \leq x \leq b, \\
& U(a, y)=g_{3}(y), \quad a \leq x \leq b, \quad U(b, y)=g_{4}(x), \quad a \leq y \leq b,
\end{aligned}
$$

where $\alpha$ is a non-negative constant and $f(x, y)$ is a function with sufficient smoothness. To enable in formulating the full-, half-, and quarter-sweep sweep triangle element approximation equations for problem (1), our next discussion will focus on uniform node points only as shown in Figure 1. Based on the Figure 1, the solution domain needs to be discretized uniformly in both $x$ and $y$ directions with a mesh size, $h$ which is defined as

$$
\Delta x=\Delta y=h=\frac{b-a}{n}, \quad m=n+1 .
$$

The full-, half-, and quarter-sweep networks of triangle finite elements need to be built as a guideline to derive triangle finite element approximation equations. Then these approximation equations will be used to form systems of finite element approximation equations for problem (1). On the contrary, using the same concept of the half-, and quarter sweep iterations applied to finite difference methods [7, 9], finite element networks will consist of several triangle elements in which each triangle element will involve three solid node points only of type • as depicted in Figure 1. Consequently, the implementation of the full-, half-, and quarter-sweep iterative algorithms are performed onto the node points of the same type until the iterative convergence criterion will be satisfied. Then other approximate solutions at remaining node points (i.e., points of types $\square$ and o) will be calculated directly [8, 10, 11].

The remainder of this paper is organized as follows. Section 2 discusses the finite element method based on Galerkin scheme for discretizing problem (1). 


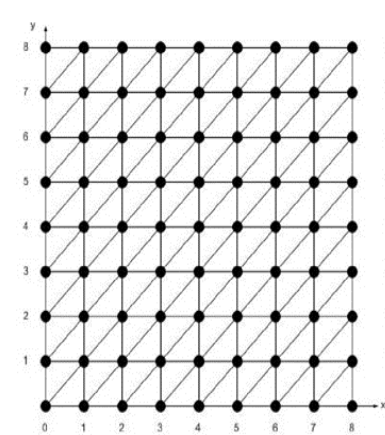

a).

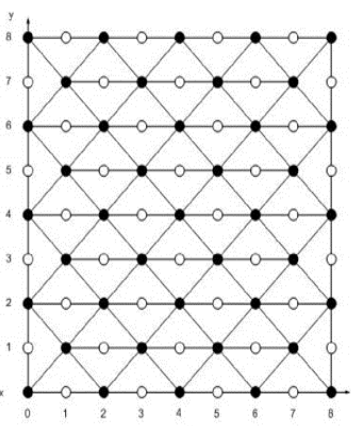

b).

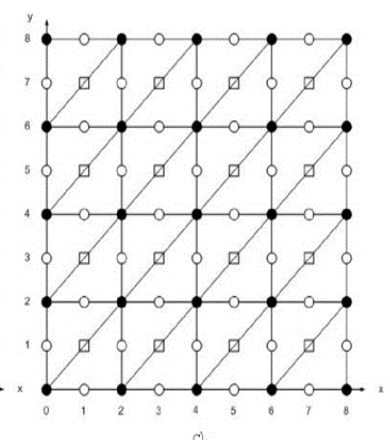

c)

Figure 1: a). b). and c). show the distribution of uniform node points for the full-, half-, and quarter-sweep cases respectively at $n=8$.

Section 3 presents the formulation and implementation of the family of GS methods in solving linear systems generated from the triangle element approximation equations. Section 4 presents some numerical examples, while conclusions are presented in Section 5.

\section{Quarter-Sweep Triangle Element Approximations}

As explained in the previous section, discretization based on the Galerkin scheme was used to construct approximation equations for problem (1) by using the first order triangle finite element approximation equation. Considering three node points $\bullet$ only, the general approximation of the function, in the form of interpolation function for an arbitrary triangle element, $e$ is given by $[1,2,5]$

$$
\widetilde{U}^{[e]}(x, y)=N_{1}(x, y) U_{1}+N_{2}(x, y) U_{2}+N_{3}(x, y) U_{3}
$$

and the shape functions $N_{k}(x, y), k=1,2,3$, can generally be shown as:

$$
N_{k}(x, y)=\frac{1}{|A|}\left(a_{k}+b_{k} x+c_{k} y\right), \quad k=1,2,3
$$

where

$$
\begin{gathered}
|A|=x_{1}\left(y_{2}-y_{3}\right)+x_{2}\left(y_{3}-y_{1}\right)+x_{3}\left(y_{1}-y_{2}\right), \\
{\left[\begin{array}{l}
a_{1} \\
a_{2} \\
a_{3}
\end{array}\right]=\left[\begin{array}{l}
x_{2} y_{3}-x_{3} y_{2} \\
x_{3} y_{1}-x_{1} y_{3} \\
x_{1} y_{2}-x_{2} y_{1}
\end{array}\right],\left[\begin{array}{l}
b_{1} \\
b_{2} \\
b_{3}
\end{array}\right]=\left[\begin{array}{l}
y_{2}-y_{3} \\
y_{3}-y_{1} \\
y_{1}-y_{2}
\end{array}\right],\left[\begin{array}{l}
c_{1} \\
c_{2} \\
c_{3}
\end{array}\right]=\left[\begin{array}{l}
x_{3}-x_{2} \\
x_{1}-x_{3} \\
x_{2}-x_{3}
\end{array}\right],}
\end{gathered}
$$


Furthermore, the first order partial derivatives of the shape functions towards $x$ and $y$ can be shown, respectively, as follows:

$$
\left.\begin{array}{rl}
\frac{\partial}{\partial x}\left(N_{k}(x, y)\right) & =\frac{b_{k}}{\operatorname{det} A} \\
\frac{\partial}{\partial y}\left(N_{k}(x, y)\right) & =\frac{c_{k}}{\operatorname{det} A}
\end{array}\right\}, \quad k=1,2,3 .
$$

Based on the definition of the hat function $[5,6,12], R_{r, s}(x, y)$, the approximation function, in case of the full-, half- and quarter-sweep cases for the entire domain in Eq. (1), is abridged, respectively, as follows:

$$
\begin{aligned}
& \widetilde{U}(x, y)=\sum_{r=0}^{m} \sum_{s=0}^{m} R_{r, s}(x, y) U_{r, s} \\
& \widetilde{U}(x, y)=\sum_{r=0,2,4}^{m} \sum_{s=0,2,4}^{m} R_{r, s}(x, y) U_{r, s}+\sum_{r=1,3,5}^{m-1} \sum_{s=1,3,5}^{m-1} R_{r, s}(x, y) U_{r, s}
\end{aligned}
$$

and

$$
\widetilde{U}(x, y)=\sum_{r=0,2,4}^{m} \sum_{s=0,2,4}^{m} R_{r, s}(x, y) U_{r, s} .
$$

In a similar manner, the definition of the approximate function, $f(x, y)$ for all cases can be easily defined via the hat function. In fact, Eqs. (6), (7) and (8) can be denoted as approximate solutions for problem.

To construct the full-, half-, and quarter-sweep linear finite element approximation equations for problem (1), the constructions of approximation equations in this study are limited by taking account of the Galerkin scheme. Now consider the Galerkin residual method $[13,14]$ to be given as follows:

$$
\iint_{D} R_{i, j}(x, y) E_{i, j}(x, y)=0, \quad i, j=0,1,2, \ldots, m,
$$

where $E(x, y)=\frac{\partial^{2} U}{\partial x^{2}}+\frac{\partial^{2} U}{\partial y^{2}}-\alpha U-f(x, y)$ is a residual function. By applying the boundary conditions, Eq. (9) can be shown in the following form:

$$
\begin{aligned}
& \int_{\lambda}\left(-R_{i, j}(x, y) \frac{\partial u}{\partial y} d x+R_{i, j}(x, y) \frac{\partial u}{\partial x} d y-\alpha R_{i, j}(x, y) U\right) \\
& -\int_{a}^{b} \int_{a}^{b}\left(\frac{\partial R_{i, j}(x, y)}{\partial x} \frac{\partial u}{\partial x}+\frac{\partial R_{i, j}(x, y)}{\partial y} \frac{\partial u}{\partial y}+\alpha R_{i, j}(x, y) U\right) d x d y=F_{i, j},
\end{aligned}
$$


where

$$
F_{i, j}=\int_{a}^{b} \int_{a}^{b} R_{i, j}(x, y) f(x, y) d x d y .
$$

By replacing Eq. (5) and imposing the boundary conditions into problem (1), Eq. (10) generates a linear system for all cases. For clarity, the linear system can generally be presented as follows:

$$
\begin{aligned}
& -\sum \sum K_{i, j, r, s}^{*}=\sum \sum C_{i, j, r, s}^{*}, \\
& -\sum \sum L_{i, j, r, s}^{*}=\sum \sum D_{i, j, r, s}^{*},
\end{aligned}
$$

where,

$$
\begin{gathered}
K_{i, j, r, s}^{*}=\int_{a}^{b} \int_{a}^{b}\left(\frac{\partial R_{i, j}}{\partial x} \frac{\partial R_{r, s}}{\partial x}\right) d x d y+\int_{a}^{b} \int_{a}^{b}\left(\frac{\partial R_{i, j}}{\partial y} \frac{\partial R_{r, s}}{\partial y}\right) d x d y \\
L_{i, j, r, s}^{*}=\alpha \int_{a}^{b} \int_{a}^{b} R_{i, j}(x, y) R_{r, s}(x, y) d x d y \\
C_{i, j, r, s}^{*}=\int_{a}^{b} \int_{a}^{b}\left(R_{i, j}(x, y) R_{r, s}(x, y)\right) d x d y \\
D_{i, j, r, s}^{*}=\alpha \int_{a}^{b} \int_{a}^{b}\left(R_{i, j}(x, y) R_{r, s}(x, y)\right) d x d y .
\end{gathered}
$$

In essence, the linear system in Eqs. (11) and (12) for the full-, half- and quarter-sweep cases can be straightforwardly expressed in stencil form, respectively, as follows:

Full-sweep:

$$
\left[\begin{array}{lll} 
& \beta_{2} & \beta_{3} \\
\beta_{2} & \rho_{1} & \beta_{2} \\
\beta_{3} & \beta_{2} &
\end{array}\right] U_{i, j}=\beta_{1}\left[\begin{array}{lll} 
& 1 & 1 \\
1 & 6 & 1 \\
1 & 1 &
\end{array}\right] f_{i, j}
$$

where 


$$
\beta_{1}=h^{2} / 12, \rho_{1}=-\left(4+\alpha 6 \beta_{1}\right), \beta_{2}=1-r \beta_{1}, \beta_{3}=r \beta_{1} .
$$

Half-sweep:

$$
\begin{aligned}
& {\left[\begin{array}{cccc}
\beta_{5} & & \beta_{5} & \\
& \rho_{2} & & \beta_{6} \\
\beta_{5} & & \beta_{5} &
\end{array}\right] U_{i, j}=\beta_{4}\left[\begin{array}{cccc}
1 & & 1 & \\
& 5 & & 1 \\
1 & & 1 &
\end{array}\right] f_{i, j}, \quad i=1,} \\
& {\left[\begin{array}{ccccc} 
& \beta_{5} & & \beta_{5} & \\
\beta_{6} & & \rho_{2} & & \beta_{6}
\end{array}\right] U_{i, j}=\beta_{4}\left[\begin{array}{ccccc} 
& 1 & & 1 & \\
& \beta_{5} & & \beta_{5} &
\end{array}\right] f_{i, j}, \quad i \neq 1, n,} \\
& {\left[\begin{array}{cccc} 
& \beta_{5} & & \beta_{5} \\
\beta_{6} & & \rho_{2} & \\
& \beta_{5} & & \beta_{5}
\end{array}\right] U_{i, j}=\beta_{4}\left[\begin{array}{cccc} 
& 1 & & 1 \\
1 & & 5 & \\
& 1 & & 1
\end{array}\right] f_{i, j}, \quad i=n,}
\end{aligned}
$$

where

$$
\beta_{4}=h^{2} / 6, \rho_{2}=-\left(4+\alpha 6 \beta_{4}\right), \beta_{5}=1-r \beta_{4}, \beta_{5}=r \beta_{4} .
$$

Quarter-sweep:

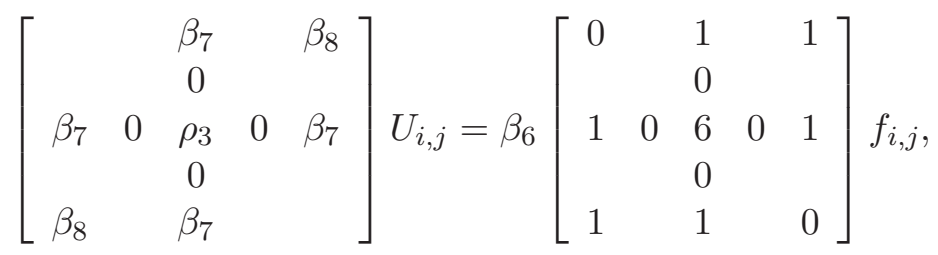

where

$$
\beta_{6}=4 h^{2} / 12, \rho_{3}=-\left(4+\alpha 6 \beta_{6}\right), \beta_{7}=1-r \beta_{6}, \beta_{8}=r \beta_{6} .
$$

As a matter of fact, the stencil forms in Eqs. (13) to (15) implicate seven node points in articulating their approximation equations. Then, by taking description of these computational molecules, the further discussion in the next section for the family of GS methods will be forthright to explain the manner to construct their formulation. 


\section{Formulation of Family of GS Methods}

To derive the formulation of family of GS methods, let Eqs. (13) to (15) be represented in a generalized form as follows:

$$
A U=f,
$$

where $A, U$ and $f$ indicate the coefficients matrix, unknown vector and known vector respectively.

As afore-mentioned, the GS method family consists of FSGS, HSGS and QSGS iterative methods, which are applied to solve the corresponding finite element approximate equations, in Eqs. (11) and (12). In what follows we consider the linear system (16) where the matrix $A$ has Property $A^{(\pi)}$ and $\pi$-consistently ordered. Thus we present some definitions, see [3].

Definition 1. Let $A$ be a real matrix. Then, $A=M-N$ is referred to as

1. a regular splitting if $M$ is nonsingular, $M^{-1} \geqslant 0$ and $N \geqslant 0$.

2. a weak regular splitting if $\mathrm{M}$ is nonsingular, $M^{-1} \geqslant 0$ and $N \geqslant 0$.

3. a nonnegative splitting, if $M^{-1} \geqslant 0$.

4. a convergent splitting, if $\rho\left(M^{-1} N\right)<1$.

Based on regular splitting, the GS splitting can be dened as follows:

Definition 2. Let $A=D-L-V$, where $D,-L$ and $-V$ are diagonal, strictly lower triangular and strictly upper triangular parts of matrices $A$ respectively. We call $A=M-N$ the GS splitting of $A$, if $M=D-L$ and $N=V$. In addition, the splitting is called

1. GS convergent $\rho\left(M^{-1} N\right)<1$.

2. GS reguler if $M^{-1}=\geqslant 0$ and $N \geqslant 0$.

3. a nonnegative splitting, if $M^{-1} \geqslant 0$.

4. a convergent splitting, if $M^{-1}=(D-L)^{-1} \geqslant 0$ and $N=V \geqslant 0$.

Hence, the common scheme for all three GS methods can be written as follows:

$$
U^{(k+1)}=(D-L)^{-1}\left(V U^{(k)}+f\right) \text {. }
$$


Actually, the iterative methods attempt to find a solution to the system of linear equations by iteratively solving the linear system using approximations for the vector $U$. Iterations continue until the solution is within a predetermined acceptable bound on the error [3]. However, our cases will be restricted on the formulation of QSGS method. By determining values of matrices $D,-L$ and $-V$, as stated in Definition 3.2, the general algorithm for QSGS method with quarter-sweep finite element approximate equations, respectively, to solve problem (1) would generally be described as follows:

$$
\begin{array}{r}
U_{i, j}^{(k+1)}=\frac{1}{\rho_{3}}\left(\beta_{7}\left(U_{i+2, j}^{(k)}+U_{i-2, j}^{(k)}+U_{i, j+2}^{(k)}+U_{i, j-2}^{(k)}\right)\right. \\
\left.-\beta_{8}\left(U_{i+2, j+2}^{(k)}+U_{i-2, j-2}^{(k)}\right)-F_{i, j}\right),
\end{array}
$$

where

$$
F_{i, j}=\beta_{6}\left(f_{i-2, j}+f_{i+2, j}+f_{i, j-2}+f_{i, j+2}+f_{i-2, j-2}+f_{i+2, j+2}+6 f_{i, j}\right) .
$$

Eq. (18) allows us to iterate through quarter of the points, lying on the $2 h$-grid. Again, it can be observed that Eq. (18) involves points of type •. Therefore, the iteration can be carried out autonomously involving only this type of point. Hence the QSGS method is defined as,

1. Discretize the solution domain into point of types (ie., •) as shown in Figure 1(c).

2. Perform iterations (using Eqs. (18)).

3. Evaluate the solutions at the remaining points.

a) points of type $\square$ using Eq. (14).

b) points of type $\circ$ using Eq. (13).

4. Display approximate solutions.

\section{Numerical Results}

In order to compare the performances of the iterative methods described in the previous section, several numerical experiments were carried out on the following two Helmholtz equations.

Example 1. ([11]) 


$$
\begin{gathered}
\frac{\partial^{2} U}{\partial x^{2}}+\frac{\partial^{2} U}{\partial y^{2}}=\left(x^{2}+y^{2}\right) e^{x y}-\alpha e^{x y}, \\
U(x, 0)=1, \quad U(x, 1)=e^{x} \\
U(0, y)=1, \quad U(1, y)=e^{y} .
\end{gathered}
$$

and the exact solution of Example 1 is given by

$$
U(x, y)=e^{x y} .
$$

Example 2. ([11])

$$
\begin{gathered}
\frac{\partial^{2} U}{\partial x^{2}}+\frac{\partial^{2} U}{\partial y^{2}}-\alpha U=-(\cos (x+y)+\cos (x-y))-\alpha \cos (x) \cos (y), \\
U(x, 0)=\cos x, \quad U\left(x, \frac{\pi}{2}\right)=0 \\
U(0, y)=\cos y, \quad U(\pi, y)=-\cos y .
\end{gathered}
$$

and the exact solution of Example 2 is given by

$$
U(x, y)=\cos (x) \cos (y) .
$$

All the simulations were implemented by a computer with processor Intel(R) Core (TM) i7 CPU 860@2.80Ghz with memory is 6.00GB. Throughout the simulations, the convergence test is considered the tolerance error $\varepsilon=10^{-10}$ and carried out on several different mesh sizes such as 284, 308, 332 and 356. In comparison, the FSGS method acts as the control of comparison of numerical results. Three criteria will be considered for methods such as the number of iterations (k), execution time (t) and its maximum and absolute error (Abs. Error) in comparison.

The results of numerical simulations, which were obtained from implementations of the FSGS, HSGS and QSGS methods for Examples 1 and 2, have been tabulated in Table 1 respectively. In the meantime, reduction percentages in terms of number of iterations and computational time for HSGS and QSGS methods compared with FSGS method have been tabulated in Table 2. 
Table 1: The numerical simulations of the three methods for Examples 1 and 2 .

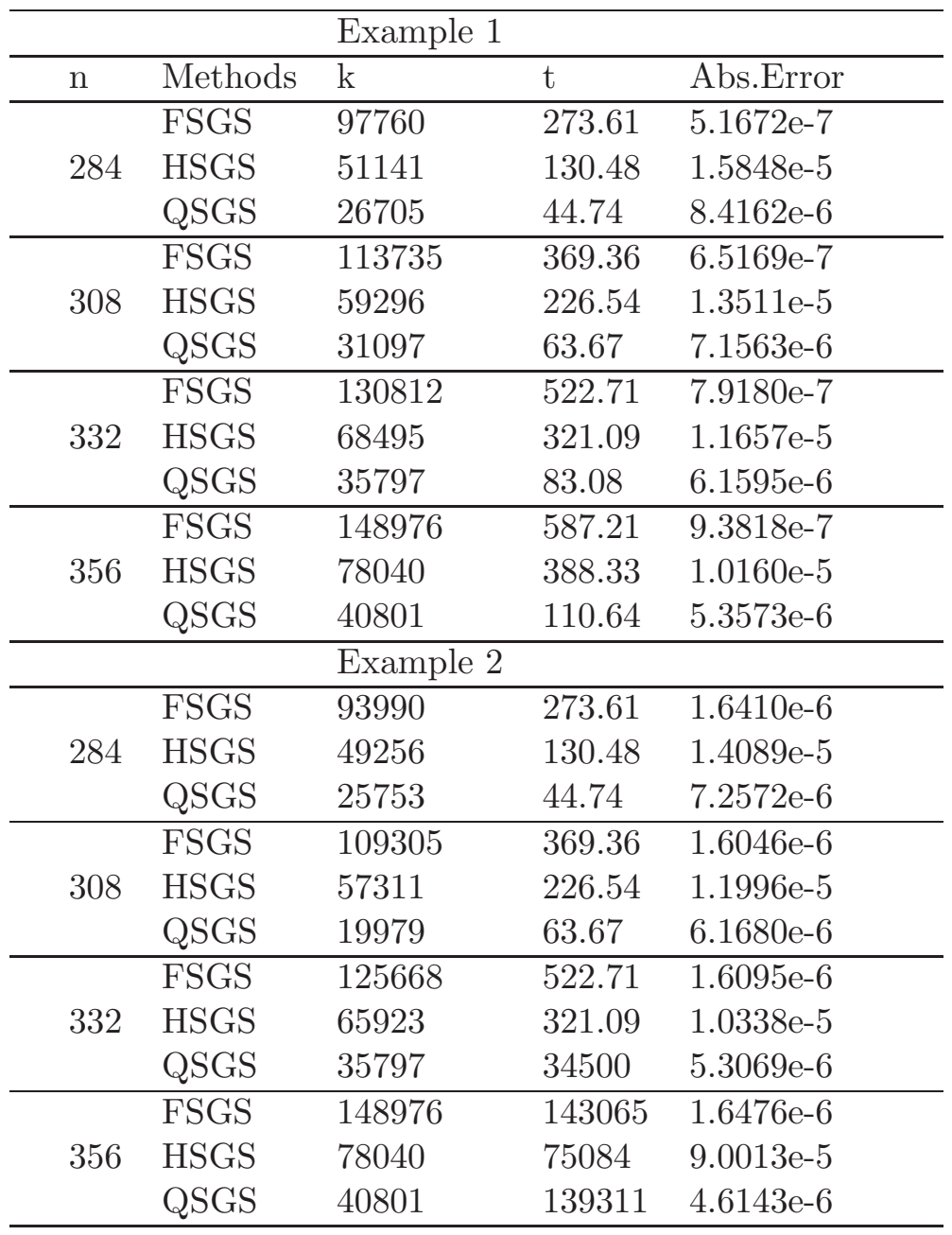

\section{Conclusions}

In this paper, we have presented an application of the quarter-sweep iterations with a GS method for solving sparse linear systems generated from the first order triangle finite element approximation equation by using the Galerkin scheme.

Overall, the numerical results show that the QSGS method is a better 
Table 2: : The reduction percentages of the HSGS and QSGS methods compared with FSGS method.

\begin{tabular}{lll}
\hline \multicolumn{3}{l}{ Example 1 } \\
\hline Methods & $\mathrm{k}$ & $\mathrm{t}$ \\
\hline HSGS & $47.69-49.63$ & $75.40-77.27$ \\
QSGS & $72.61-72.68$ & $81.16-83.65$ \\
\hline \multicolumn{3}{c}{ Example 2 } \\
\hline Methods & $\mathrm{k}$ & $\mathrm{t}$ \\
\hline HSGS & $47.55-47.63$ & $47.94-54.68$ \\
QSGS & $75.52-72.60$ & $89.49-89.72$ \\
\hline
\end{tabular}

method compared to the HSGS, and FSGS methods in the sense of number of iterations and execution time. This is mainly because of the reduction in term of computational complexity; since the implementations of the QSGS will only consider approximately quarter of all interior node points in a solution domain.

\section{References}

[1] C.A.J. Fletcher, The Galerkin method: An introduction. In: J. Noye, (pnyt.), Numerical Simulation of Fluid Motion, North-Holland Publishing Company, Amsterdam (1978), 113-170.

[2] C.A.J. Fletcher, Computational Galerkin Method, Springer Ser. in Computational Physics, Springer-Verlag, New York (1984).

[3] D.M. Young, Iterative Solution of Large Linear Systems, Academic Press, London (1971).

[4] G. Yagawa, T. Furukawa, Recent developments of Free Mesh method, Int. J. for Numer. Meth. in Engi., 47 (2000), 1419-1443.

[5] D.J. Evans, and M.M. Martins, The AOR method for $A X-X B=C$, Int. J. of Comp. Math., 52 (1994), 75-82.

[6] J. Sulaiman. M.K. Hasan, and M. Othman, In: Red-Black Half-Sweep Iterative Method Using Triangle Finite Element Approximation for $2 D$ Poisson Equations, LNCS 4487, Springer-Verlag, Berlin (2007), 326-333. 
[7] J. Sulaiman, M.K. Hasan, M. Othman, In: Red-Black EDGSOR Iterative Method Using Triangle Element Approximation for 2D Poisson Equations, LNCS 4707, Springer-Verlag, Berlin (2007), 298-308.

[8] M.K.M. Akhir, M. Othman, J. Sulaiman, Z.A. Majid, and M. Suleiman, Half-sweep modified successive over-relaxation for solving two-dimensional Helmholtz equations, Aust. J. of Bas. and App. Scie., 12 (2011), 30333039 .

[9] M.K.M. Akhir, M. Othman, J. Sulaiman, Z.A. Majid, and M. Suleiman, Half sweep iterative method for solving two-dimensional Helmholtz equations, Int. J. of App. Math. and Stat., 29 (2012), 101-109.

[10] M.K.M. Akhir, M. Othman, J. Sulaiman, Z.A. Majid, and M. Suleiman, An efficient quarter-sweep modified successive over-relaxation for solving two-dimensional Helmholtz equations, Int. J. of Pure and Appl. Math., 80 (2012), 751-764.

[11] M.K.M. Akhir, J. Sulaiman, HSGS method for the finite element solution of two-dimensional Helmholtz equations, Global J. of Math., 40 (2015), 367-373.

[12] O.C. Zienkiewicz, Why finite elements?, In: R.H. Gallagher, J.T. Oden, C.Taylor, O.C. Zienkiewicz (Eds), Finite Elements In Fluids Volume, John Wiley and Sons, London (1975).

[13] P.E. Lewis, J.P. Ward, The Finite Element Method: Principles and Applications, Addison-Wesley Publishing Company, Wokingham, 1991.

[14] R. Vichnevetsky, Computer Methods for Partial Differential Equations, Vol. I, Prentice-Hall, New Jersey (1981).

[15] T. Belytschko, Y. Krongauz, D. Organ, M. Fleming, and P. Krysl, Mesh less methods: An overview and recent developments, Comp. Meth. in App. Mech. and Engi., 139 (1996), 3-47.

[16] T. Zhu, A new meshless regular local boundary integral equation (MRLBIE) approach, Int. J. for Num. Meth. in Eng., 46 (1999), 1237-1252. 